\title{
Tumores malignos en pacientes adultos, diagnosticados por primera vez en el Hospital ISSSTEP durante el 2017
}

\section{Solid malignant tumors in adults, with the first diagnosis during 2017 in the ISSSTEP hospital}

\author{
Cyntia Cruz-García ${ }^{1}$, Ixel Escamilla-López ${ }^{1,2 *}$, Alfredo Márquez-Melgarejo ${ }^{3}$, Andrea Castro-Sánchez ${ }^{1,2}$ y \\ Eduardo Téllez-Bernal',2
}

${ }^{1}$ Unidad Médica Onco-Hematológica de Puebla; ${ }^{2}$ Departamento de Oncología, Hospital de Especialidades, Instituto de Seguridad y Servicios Sociales de los Trabajadores al Servicio de los Poderes del Estado de Puebla; ${ }^{3}$ Departamento de Patología, Hospital de Especialidades, Instituto de Seguridad y Servicios Sociales de los Trabajadores al Servicio de los Poderes del Estado de Puebla. Puebla, Pue., México

\section{Resumen}

Introducción: Diversas instituciones mundiales, nacionales y locales, como el Instituto de Seguridad y Servicios Sociales de los Trabajadores al Servicio de los Poderes del Estado de Puebla (ISSSTEP), buscan constantemente obtener cifras efectivas sobre los alcances del cáncer en nuestra sociedad actual, los cuales demuestran que el cáncer es la principal causa de muerte en todo el mundo. Objetivo: Describir los tumores malignos no hematológicos diagnosticados durante el 2017 en el Hospital de Especialidades ISSSTEP. Material y métodos: Se realizó la revisión de la base de datos del departamento de patología del Hospital ISSSTEP de tumores sólidos no hematológicos en mayores de 18 años; se recabaron datos de edad, sexo, sitio de tumor primario y tipo histológico de cada caso y se realizó estadística descriptiva básica. Resultados: Durante el 2017 se diagnosticaron 326 casos de tumores malignos no hematológicos en nuestra institución; el 59.2\% correspondió al sexo femenino. Los tumores de piel, mama, próstata, colorrectales y el cáncer de tiroides ocuparon los cinco primeros lugares durante este periodo, representado el $59.7 \%$ de todos los casos. Conclusión: El número de casos de cáncer se incrementa de forma paulatina, asociado a diversos factores. En nuestra población se demostró un mismo comportamiento, manteniendo al cáncer de mama y de próstata como los tumores malignos que afectan mayormente a mujeres y hombres.

Palabras clave: Cáncer. Estadística. Mama. Próstata.

\begin{abstract}
Introduction: Different public health institutions had tried to describe the cancer global impact because it represents the more important cause of death worldwide. Objective: To describe the prevalence of malignant solid tumors during 2017 in the ISSSTEP hospital. Materials and methods: We retrospectively analyzed the Pathology Department database to identify malignant solid tumors diagnosed during 2017 in the ISSSTEP hospital. The solid tumors were classified according to the patient's sex and age at diagnosis, and the tumor anatomic site and histologic type. Results: In 2017, 326 solid tumors were diagnosed, 59.2\% in women. Malignant skin tumors, breast cancer, prostate cancer, colorectal cancer and thyroid cancer were the five most prevalent tumor types, representing 59.7\% of all tumors. Conclusion: Breast cancer and prostate cancer are the leading causes of cancer by sex in the ISSSTEP hospital, consistent with the national statistics for Mexico.
\end{abstract}

Key words: Cancer. Statistics. Breast. Prostate.

Correspondencia:

*Ixel Escamilla López

E-mail: ixel.escamilla @gmail.com
Fecha de recepción: 10-09-2020

Fecha de aceptación: 19-10-2021

DOI: 10.24875/RHJM.20000109
Disponible en internet: 17-12-2021 Rev Hosp Jua Mex. 2021;88(4):181-186

www.revistahospitaljuarez.com 1405-9622/C 2021 Sociedad Médico-Quirúrgica del Hospital Juárez de México, A.C. Publicado por Permanyer. Este es un artículo open access bajo la licencia CC BY-NC-ND (http://creativecommons.org/licenses/by-nc-nd/4.0/). 


\section{Introducción}

La incidencia mundial de neoplasias malignas ha experimentado un incremento significativo en los últimos años. Según la Organización Mundial de la Salud (OMS), en 2015 las enfermedades malignas de diversos tipos no solo se han incrementado, sino que representan la segunda causa de muerte en el mundo antes de los 70 años. En ese mismo año ocasionó 8.8 millones de defunciones, identificándose cinco tipos de cáncer responsables del mayor número de fallecimientos: cáncer pulmonar (1.69 millones de muertes), cáncer hepático (788 mil defunciones), cáncer colorrectal (774 mil muertes), cáncer gástrico (754 mil defunciones) y cáncer de mama (571 mil muertes)'

La base de datos del Global Cancer Observatory (GLOBOCAN) en su última actualización reporta 18.1 millones de casos de cáncer a nivel mundial, lo que representa 4 millones de casos nuevos más que en su actualización previa. A su vez esta plataforma hace una predicción estimada de 11.4 millones de casos nuevos para el 2040².

La combinación del incremento poblacional, urbanización, cambios en la dieta y aumento de la expectativa de vida impulsan el marcado aumento en la incidencia de neoplasias malignas en países en vías de desarrollo. En nuestro país, el cáncer se ubica como la tercera causa de muerte, después de diabetes y de las enfermedades hipertensivas ${ }^{3}$.

En el año 2017 el Instituto de Seguridad Social al Servicio de los Trabajadores del Estado de Puebla (ISSSTEP) registró una población de 158,419 derechohabientes, lo cual representa el $2.6 \%$ de la población total del Estado de Puebla. En este año también se reportaron dentro de la institución 256 defunciones hospitalarias, de las cuales 23 fueron secundarias a neoplasias malignas, dejando a este padecimiento como el responsable del $8.9 \%$ de las defunciones ${ }^{4}$.

\section{Objetivos}

Presentar información institucional actualizada de los tumores malignos sólidos diagnosticados en mayores de 18 años en el Hospital ISSSTEP durante el 2017 con la finalidad de crear una base de datos que ayude a la detección, diagnóstico y tratamiento oportunos de los principales tipos de cáncer.

\section{Material y metodología}

Se realizó la revisión de base de datos del departamento de patología del instituto de todos los casos de tumores malignos diagnosticados en el 2017, con lo que se creó una base de datos en el departamento de oncología, clasificando los casos de acuerdo con el sitio anatómico del tumor primario, el tipo y subtipo histológico del tumor analizado, así como edad y sexo del paciente. Se obtuvieron datos pronósticos patológicos específicos de acuerdo con el tumor diagnosticado.

Se incluyeron todos los pacientes mayores de 18 años con diagnóstico de tumor maligno sólido. Se excluyeron los pacientes menores de 18 años, los casos de recurrencia de la enfermedad, así como casos de reportes de biopsias de metástasis con diagnóstico inicial en años previos.

Se obtuvieron frecuencias relativas de los tumores diagnosticados durante el 2017 y su tendencia en el año de estudio.

El presente estudio fue aprobado por el comité de ética e investigación del instituto. Debido a que el estudio no tuvo involucro directo de los pacientes (sin revelar información personal) y al representar nulo riesgo a los participantes, no se requirió consentimiento informado.

\section{Resultados}

Durante el 2017, en el Hospital ISSSTEP se reportaron un total de 326 casos de tumores sólidos malignos no hematológicos (Tabla 1), correspondiendo 193 casos al sexo femenino (59.20\%) y 133 al masculino $(40.80 \%)$.

El cáncer de piel resultó ser el tumor maligno más frecuente, con 55 casos (16.9\%), de los cuales el $63.63 \%(n=35)$ corresponden al sexo femenino. La media de edad al diagnóstico fue de 71.7 años (rango: 34-87). El tipo histológico más frecuente correspondió en el $67.3 \%$ de los casos $(n=37)$ al tipo basocelular, en el $18.2 \%(n=10)$ al epidermoide (carcinoma de células escamosas) y en el $7.3 \%$ a melanoma $(n=4)$; de estos, el principal subtipo histológico correspondió al acral lentiginoso $(n=3)$, seguido del amelánico $(n=1)$. Se reportaron también otros tipos histológicos de cáncer de piel: mixto con histología basocelular y epidermoide, sebáceo y porocarcinoma.

El cáncer de mama se coloca como el segundo tumor maligno más frecuente, con 52 casos (15.9\%), presentándose la totalidad de los casos en el sexo femenino, con media de edad de 56.6 años (rango: 25-91). En cuanto al tipo histológico, el carcinoma ductal fue el más diagnosticado $(n=48)$, seguido del lobulillar y el mixto, con dos casos cada uno. El análisis por 
Tabla 1. Incidencia de cáncer en el Instituto de Seguridad y Servicios Sociales de los Trabajadores al Servicio de los Poderes del Estado de Puebla en el 2017

\begin{tabular}{|c|c|c|c|c|}
\hline Tumor & n (\%) & $\begin{array}{l}\text { Edad media } \\
\text { (rango) }\end{array}$ & Hombre & Mujer \\
\hline Piel & $55(16.6 \%)$ & 71.7 (34-87) & 20 & 35 \\
\hline Mama & $52(15.7 \%)$ & $56.6(25-91)$ & 0 & 52 \\
\hline Próstata & $42(12.6 \%)$ & $7.4(55-87)$ & 42 & 0 \\
\hline Colorrectal & $24(7.25 \%)$ & 61.7 (33-79) & 15 & 9 \\
\hline Tiroides & $22(6.6 \%)$ & $45.6(18-84)$ & 4 & 18 \\
\hline Riñón & $16(5.1 \%)$ & $58.2(38-85)$ & 10 & 6 \\
\hline $\begin{array}{l}\text { Cuerpo } \\
\text { uterino }\end{array}$ & $15(4.2 \%)$ & $56.2(29-77)$ & 0 & 15 \\
\hline Estómago & $15(4.5 \%)$ & $67.1(24-91)$ & 8 & 7 \\
\hline $\begin{array}{l}\text { Cuello } \\
\text { uterino }\end{array}$ & $14(4.8)$ & 48 (29-79) & 0 & 14 \\
\hline Ovario & $10(3.0 \%)$ & $52.3(21-73)$ & 0 & 10 \\
\hline Pulmón & $10(4.2 \%)$ & $56.9(48-79)$ & 3 & 7 \\
\hline Urotelial & $7(2.1 \%)$ & $64.2(51-81)$ & 6 & 1 \\
\hline $\begin{array}{l}\text { Tejido } \\
\text { conectivo }\end{array}$ & $6(1.8 \%)$ & $53.3(44-72)$ & 3 & 3 \\
\hline $\begin{array}{l}\text { Cabeza y } \\
\text { cuello }\end{array}$ & $5(1.5 \%)$ & 77.8 (58-92) & 3 & 2 \\
\hline $\begin{array}{l}\text { Primario } \\
\text { desconocido }\end{array}$ & $5(1.5 \%)$ & 71 (58-79) & 1 & 4 \\
\hline Misceláneos & $5(1.5 \%)$ & $67.3(27-89)$ & 4 & 1 \\
\hline SNC & $4(1.2 \%)$ & $59.2(52-76)$ & 3 & 1 \\
\hline $\begin{array}{l}\text { Ámpula de } \\
\text { Vater }\end{array}$ & $4(1.2 \%)$ & 71 (66-76) & 2 & 2 \\
\hline $\begin{array}{l}\text { Vesícula } \\
\text { biliar }\end{array}$ & $3(0.9 \%)$ & $60.3(49-70)$ & 0 & 3 \\
\hline Testículo & $3(0.9 \%)$ & $41.3(32-53)$ & 3 & 0 \\
\hline Hígado & $3(0.9 \%)$ & $69(60-76)$ & 2 & 1 \\
\hline $\begin{array}{l}\text { Intestino } \\
\text { delgado }\end{array}$ & $2(0.6 \%)$ & $65(59-71)$ & 2 & 0 \\
\hline Esófago & $2(0.6 \%)$ & 73 (72-74) & 2 & 0 \\
\hline Vagina & $1(0.3 \%)$ & 79 & 0 & 1 \\
\hline Vulva & $1(0.3 \%)$ & 74 & 0 & 1 \\
\hline Total & 326 & $60.3(18-98)$ & 133 & 193 \\
\hline
\end{tabular}

SNC: sistema nervioso central.

inmunohistoquímica mostró 27 casos correspondientes tumores luminal A (receptor progesterona y estrógeno positivos, Her2neu negativo), 7 casos Her2neu (Her2neu positivo, receptor progesterona y estrógeno negativo), 4 casos a triple negativo (Her2neu, receptor progesterona y estrógeno negativos) y 4 casos a triple positivo.

El cáncer de próstata se sitúa en tercer lugar, con 42 casos (12.8\%), con media de edad al diagnóstico de 70.4 años (rango: 55-87). Clasificándolos por grupos de edad, se observó mayor prevalencia en los grupos de 60-69 y 70-79 años. En cuanto a su histología, el adenocarcinoma de tipo acinar fue presentado en 41 casos; la neoplasia intraepitelial de alto grado fue el otro caso reportado.

En cuarto lugar se encontró el cáncer colorrectal, con 24 casos (7.4\%), con media de edad de 61.7 años (rango: 33-79); se observó predominio del sexo masculino $(n=15)$. La histología reportó 22 casos de adenocarcinoma, 1 caso de tumor del estroma gastrointestinal (GIST) y 1 tumor neuroendocrino. Se diagnosticaron 10 casos a nivel de recto, 4 casos en colon ascendente, 3 en porción descendente, 2 en rectosigmoides, 2 en porción transversa, 1 en sigmoides y 1 en ángulo hepático.

En quinto lugar se encontró el cáncer de tiroides, con 22 casos (6.7\%), 18 de los casos en el sexo femenino. La edad media de presentación fue de 47.3 años (rango: 18-84). Todos los casos correspondieron a tumores bien diferenciados, 21 a carcinoma papilar y 1 a carcinoma folicular.

En sexto lugar se encontró el cáncer renal, con 16 casos (4.9\%), el cual tuvo predominio en el sexo masculino con 10 casos. La edad media fue de 58.2 años (rango: 38-85). La histopatología mostró 13 casos con carcinoma de células claras, 2 casos de carcinoma papilar y 1 leiomiosarcoma.

En el séptimo lugar se encontraron el cáncer gástrico y el de endometrio, con 15 casos (4.6\%) cada uno. En cuanto al cáncer gástrico, se encontraron 8 casos del sexo femenino; con una edad media de presentación de 67.8 años (rango: 24-91). En cuanto a la histopatología, 14 de los casos fueron adenocarcinoma (5 de tipo difuso con células en anillo de sello y 4 de tipo intestinal, en el resto no se especificó el subtipo) y se reportó 1 caso de GIST. De los 15 tumores gástricos, 1 fue diagnosticado en etapa IV. El cáncer de endometrio tuvo media de edad de 56.2 años (rango: 29-77). La histopatología fue en su totalidad adenocarcinoma tipo endometrioide.

El cáncer cervicouterino se posiciona como el octavo tumor más habitual, con 14 casos (4.29\%). La edad mínima de presentación fue de 29 años, mientras que la máxima fue de 98 , con una edad media de 48 . De los 
14 casos, 13 fueron de histología epidermoide (8 de los cuales fueron carcinomas in situ) y 1 adenocarcinoma.

El noveno lugar lo compartieron los tumores torácicos y el cáncer de ovario, con 10 casos cada uno (3.1\%). El cáncer de pulmón fue predominante en el sexo femenino, con 7 casos, y la edad media fue de 66.7 años (rango: 58-79). La histología reportó 6 casos de adenocarcinoma, 3 de carcinoma epidermoide y 1 mesotelioma maligno. Dos casos fueron diagnosticados en etapa IV. El cáncer de ovario tuvo una edad media de 52.3 años (rango: 21-73). La histopatología mostró 8 tumores epiteliales (4 fueron de subtipo seroso, 2 endometrioide, 1 de células claras y en otro caso no se especificó el subtipo) y dos tumores no epiteliales ( 1 tumor de células de la granulosa y 1 carcinoma intraepitelial).

El décimo lugar lo ocupó el cáncer urotelial, el cual contó con 7 casos (2.1\%) reportados. El cáncer de urotelio fue predominante en el sexo masculino, contando este con 6 casos, presentándose con edad media de 64.2 años. Dos casos correspondientes a lesiones renales y 1 a uretra masculina, el resto a lesiones de vejiga. La histopatología reportó 4 carcinomas papilares, los 3 restantes reportados como carcinomas uroteliales de alto grado.

El undécimo cáncer más frecuente fue el de tejido conectivo (1.8\%), con 3 liposarcomas (2 pleomórficos y 1 desdiferenciado), 2 sarcomas de Kaposi y 1 perineuroma. Los liposarcomas se presentaron en el sexo femenino en 2 casos, ambos sarcomas de Kaposi fueron en el sexo masculino y el perineuroma se presentó en el sexo femenino. Se obtuvo una edad media al diagnóstico de 51.8 años (rango: 27-76).

El duodécimo fue compartido por los tumores de cabeza y cuello, los tumores misceláneos y los tumores con primario desconocido, con 5 casos cada uno (1.5\%). De los tumores de cabeza y cuello, 4 correspondieron a carcinomas epidermoides (dos de laringe, uno de cavidad oral y uno de pilar amigdalino) y 1 correspondió a un leiomiosarcoma infiltrando tejidos blandos de cara. La edad media fue de 77.8 años. En cuanto a los tumores misceláneos, 2 correspondieron a carcinomas tímicos (uno resecado de mediastino y otro de bronquio), 1 leiomiosarcoma retroperitoneal, 1 carcinoma epidermoide verrucoso de pene y 1 melanoma de canal anal. En cuanto a los tumores de primario desconocido, 3 fueron carcinomas pocos diferenciados (uno en ganglio linfático, un tumor abdominal y uno de implante peritoneal), los 2 restantes fueron compatibles con adenocarcinoma, ambos de citológicos de líquido pleural.
Los tumores sólidos malignos con frecuencia menor del $1.5 \%$ fueron los tumores de testículo (2 casos de seminoma clásico y 1 tumor no seminomatoso con predominio de carcinoma embrionario), los tumores de hígado (2 carcinomas hepatocelular y 1 tumor neuroendocrino metastásico), esófago (2 epidermoides), intestino delgado (1 adenocarcinoma y 1 GIST), sistema nervioso central (2 glioblastomas multiformes, 1 oligodendroglioma y 1 astrocitoma), ámpula de Vater, carcinoma vulvar, carcinoma de vagina y tumores de vesícula biliar (Tabla 1).

\section{Discusión}

La estadística nacional y mundial coinciden en que el cáncer representa una de las principales causas de morbimortalidad en la población en general, estimando que la mitad de la población masculina y un tercio de la población femenina tendrán diagnóstico de cáncer a lo largo de su vida 5 .

A nivel nacional según GLOBOCAN el tipo de cáncer más frecuente resultó ser el de mama, con 27,283 casos reportados. Entretanto, en la población de ISSSTEP durante en 2017 se observó que el cáncer de piel es más habitual, representando el $16.9 \%$ de los casos, desplazando al cáncer de mama al segundo lugar (15.9\%); sin embargo, hay que tener en cuenta que en este reporte se consideraron todos los tipos de cáncer de piel e internacionalmente para fines estadísticos se excluye el cáncer de piel no melanoma, lo cual explica estos resultados.

Ahora bien, resulta trascendental mencionar como GLOBOCAN ha registrado un acrecentamiento en la incidencia femenina de cáncer en general, cuya cifra asciende a más de 105,000 casos; mientras que en hombres se registra una cifra de 85,000 casos, esto tan solo a nivel nacional.

Según el Instituto Nacional de Estadística y Geografía, en el $69 \%$ del total de las muertes por cáncer este fue diagnosticado en estadios avanzados, obstaculizando un tratamiento exitoso ${ }^{6}$. Entre el 30 y el $50 \%$ de los cánceres se pueden prevenir, y un alto porcentaje es curable con un diagnóstico oportuno y tratamiento adecuado; por lo que se debe mencionar la importancia de la prevención primaria en clínicas de primer contacto, con especial atención en los factores de riesgo para cada tipo de cáncer.

Al comparar estos resultados con los de años previos en nuestra institución (Fig. 1), se observó incremento en el número de casos, reportando 275 casos en el 2016 contra 326 en el 2017 (15.6\% más). También se evidenció un aumento muy marcado en los tumores de 


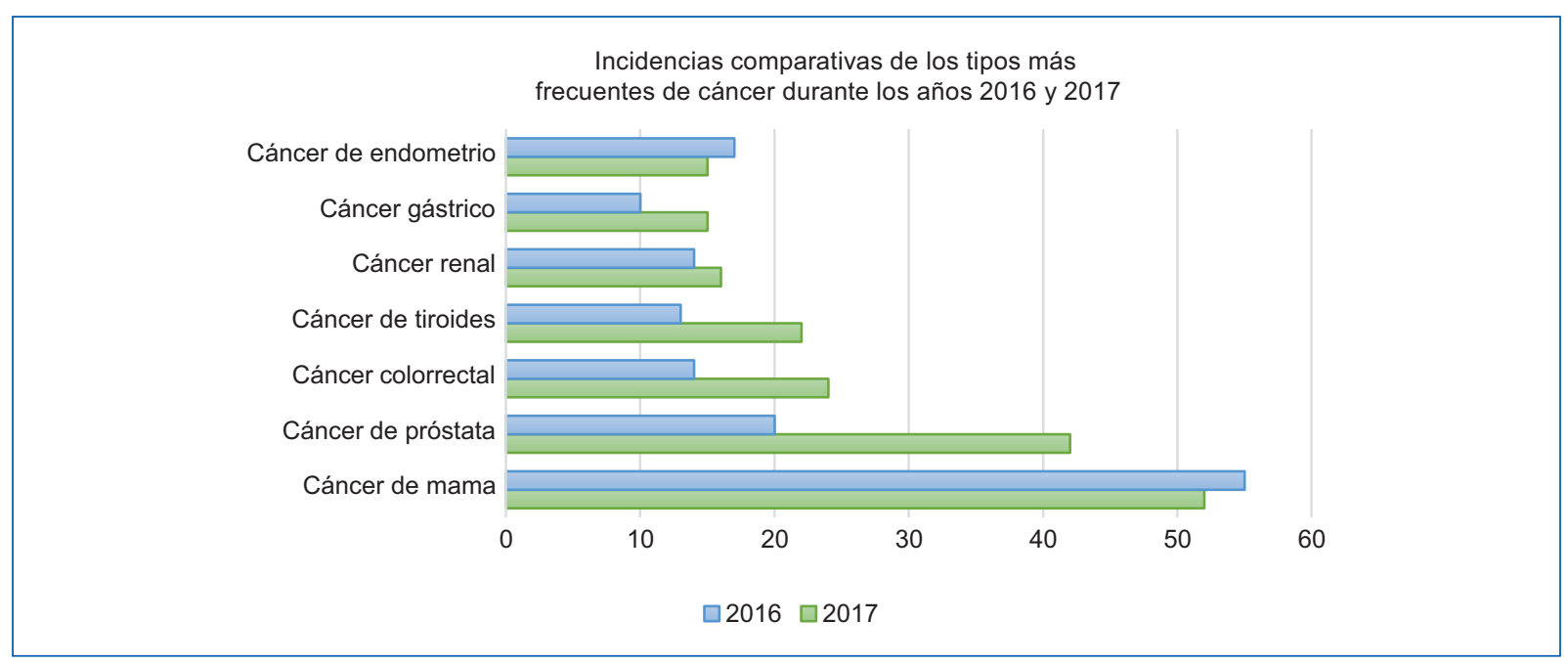

Figura 1. Comparación de incidencias de los 7 tipos más frecuentes de cáncer durante 2016 y 2017 en el hospital ISSSTEP.

próstata, con 20 casos en el 2016 contra 42 en el 2017; colorrectal con 13 casos en el 2016 y 24 en el 2017; tiroides con 13 casos en 2016 y 22 en 2017, y dos casos de cáncer de pulmón para el 2016 contra 10 casos para el 2017. Mientras que el cáncer cervicouterino, el de ovario e hígado tuvieron una disminución mínima de incidencia comparada con el 2016 (21 contra 14,11 contra 10 y 4 contra 3 , respectivamente).

Según la evidencia, se entiende que el cáncer, bajo cualquiera de sus múltiples manifestaciones, es una problemática sumamente compleja de abordar, por su carácter multifactorial. Diversos tipos de cáncer tuvieron un ritmo de aparición que ha ido en aumento en la población del ISSSTEP, siendo el 2016 el año comparador, pudiendo ser explicado por diferentes factores que deben analizarse posteriormente, como son la falta de prevención en la población estudiada o tal vez el incremento en la detección ${ }^{6}$.

El riesgo de padecer la mayoría de los tipos de cáncer se puede reducir, mediante cambios en el estilo de vida, evitando consumo de tabaco y alcohol, limitando el tiempo de exposición al sol, realizando ejercicio y manteniendo una alimentación saludable ${ }^{7}$. La prevención primaria para el cáncer de piel, mama y próstata debe situarse en las prioridades en la atención en el primer nivel de atención médica, siendo importante dotar al paciente de la información necesaria según los tipos de factores de riesgo a los que esté expuesto y los estudios de control y prevención según sea el caso del tipo de cáncer al que esté propenso para detectar la enfermedad en etapas tempranas. En general, mientras más temprano se detecte el cáncer y se comience el tratamiento, mejor calidad de vida y mayores serán las probabilidades de supervivencia.

Es importante comentar que durante la obtención de información se encontraron diferentes obstáculos para la realización de la base de datos y análisis de esta, uno de ellos fue que la mayoría de los casos no eran referidos al servicio adecuado para el tratamiento y/o seguimiento del paciente; y por parte de la población también se encontraron abandonos al tratamiento y al seguimiento médico.

\section{Conclusión}

El número de casos de cáncer se incrementa de forma paulatina, asociada a diversos factores. En nuestra población se demostró un mismo comportamiento, manteniendo al cáncer de mama y de próstata como los tumores malignos que afectan mayormente a mujeres y hombres.

\section{Agradecimientos}

Los autores agradecen al equipo de investigación, familia, amigos y pacientes del hospital.

\section{Financiamiento}

Los autores no recibieron patrocinio para llevar a cabo este artículo.

\section{Conflicto de intereses}

Los autores declaran no tener conflicto de intereses. 


\section{Responsabilidades éticas}

Protección de personas y animales. Los autores declaran que para esta investigación no se han realizado experimentos en seres humanos ni en animales.

Confidencialidad de los datos. Los autores declaran que han seguido los protocolos de su centro de trabajo sobre la publicación de datos de pacientes.

Derecho a la privacidad y consentimiento informado. Los autores declaran que en este artículo no aparecen datos de pacientes.

\section{Bibliografía}

1. Organización Mundial de la Salud. Cáncer. Nota descriptiva [Internet]. Organización Mundial de la Salud; 2017 [consultado: 13 de febrero de 2018]. Disponible en: http://www.who.int/mediacentre/factsheets/fs297/es
2. Senado de la República, Coordinación de Comunicación Social. El cáncer, problema grave de salud pública, tercera causa de muerte en México [Internet]. Gobierno de México, Senado de la República, Coordinación de Comunicación Social; 22 de marzo 2017 [consultado: 14 de febrero de 2019]. Disponible en: http://comunicacion.senado.gob.mx/index.php/informacion/boletines/35187-el-cancer-problema-grave-de-salud-publica-tercera-causa-de-muerte-en-mexico.html

3. Cancer Today [sede web]. Lyon, Francia: World Health Organization, International Agency for Research on Cancer; 2018 [consultado: 13 de febrero de 2019]. Disponible en: https://gco.iarc.fr/today

4. Gobierno de México. Defunciones hospitalarias registradas en el ISSSTEP [Internet]. Gobierno de México; 2018 [consultado: 14 de febrero del 2019]. Disponible en: http://datos.puebla.gob.mx/dataset/estadistica-epidemiologia-generada-issstep

5. Instituto Nacional del Cáncer. Estadísticas del cáncer [Internet]. EE.UU.: Instituto Nacional del Cáncer; 2018 [consultado: 25 de febrero del 2019]. Disponible en: https://www.cancer.gov/espanol/cancer/naturaleza/estadisticas

6. Instituto Nacional de Estadística y Geografía. Estadísticas a propósito del día mundial contra el cáncer [Internet]. México: Instituto Nacional de Estadística y Geografía; 2018 [consultado: 18 de marzo de 2019]. Disponible en: http://www.beta.inegi.org.mx/contenidos/saladeprensa/aproposito/2018/cancer2018_Nal.pdf

7. Secretaría de Salud. Infocáncer México: Prevenir el cáncer [Internet]. México: Secretaría de Salud; 2019 [consultado: 31 de marzo de 2019]. Disponible en: https://www.infocancer.org.mx/?c = prevenir-el-cancer 